\title{
Towards Model-Based Support for Managing Organizational Transformation
}

\author{
Daniele Barone ${ }^{1}$, Liam Peyton ${ }^{2}$ Flavio Rizzolo ${ }^{2}$, Daniel Amyot ${ }^{2}$, and John \\ Mylopoulos ${ }^{3}$ \\ 1 Department of Computer Science, University of Toronto, Toronto (ON), Canada \\ barone@cs.toronto.edu \\ 2 SITE, University of Ottawa, Ottawa (ON), Canada \\ lpeyton|frizzolo|damyot@site.uottawa.ca \\ 3 DISI, University of Trento, Trento, Italy \\ jm@disi.unitn.it
}

\begin{abstract}
In an increasingly connected and dynamic world, most organizations are continuously evolving their business objectives, processes and operations through ongoing transformation and renewal, while their external environment is changing simultaneously. In such a setting, it is imperative for organizations to continuously monitor their performance and adjust when there is a need. The technology that delivers this monitoring capability is called Business Intelligence (BI), and over the years it has come to play a central role in business operations and governance. Unfortunately, there is a huge cognitive gap between the strategic business level view of goals, processes, and performance on one hand, and the technological/implementation view of databases, networks, and computational processing offered by BI tools on the other.

In this paper, we present a model-based framework for bridging this cognitive gap and demonstrate its usefulness through a case study involving organizational transformation. The business view is modeled in terms of the Business Intelligence Model (BIM), while the data collection and reporting infrastructure is expressed in terms of the Conceptual Integration Model (CIM). The case study involves a hospital implementing a strategic initiative to reduce antibiotic resistant infections.
\end{abstract}

Key words: business intelligence, model-based, data integration, organizational transformation

\section{Introduction}

In an increasingly connected and dynamic world, most organizations are continuously evolving their business objectives, processes and operations through an ongoing process of transformation and renewal. A variety of business methodologies or frameworks exist that are intended to guide an organization to improve its business processes in an incremental way [1]. Typically, strategic initiatives identify opportunities and enact change through a continuous process of monitoring and measurement to align operational performance with strategic targets. A tool-supported methodology that can integrate goals, processes and performance 
is essential to help management implement such initiatives by automating or semi-automating some of the implementation tasks [2]. In current practice, key performance indicators play a bridging role by integrating data from a variety of sources inside and outside an organization to measure how well strategic business targets are being met [3].

Unfortunately, there is a huge cognitive gap between the strategic business level view of goals, processes, and performance and the technological view of databases, networks, and computational processing needed to deliver an implementation of these concepts. The implementation is intended to offer a monitoring function for key performance indicators that determine how the organization is doing with respect to its strategic initiatives. A modeling approach is needed that can represent both the business and technological view of things, along with computational mappings that bridge the gap between the two levels and deliver ongoing monitoring and transformation.

In this paper, we present a model-based framework for organizational transformation that builds on concepts and technologies from computer science and management. The key elements of the framework are:

- A Business Intelligence Model (BIM) that represents strategic initiatives and their associated plans in terms of goals, processes, and indicators.

- A Conceptual Integration Model (CIM) that represents a conceptual view of organizational data integrated to create focused dashboards for reporting indicators used to monitor strategic initiatives.

- A mapping framework between BIM and CIM, along with corporate dashboards that link the two levels for purposes of monitoring and reporting.

A case study is used to demonstrate the workings of our proposed framework. It involves a hospital that decides to implement a strategic initiative to reduce antibiotic resistant infections.

Section 2 of this paper includes a brief introduction into BIM and CIM concepts, as well as an overview on managing organizational transformation and BI. Section 3 introduces the different phases to manage the lifecycle of an initiative. Section 4 presents a case study drawn from a strategic initiative currently underway at a large teaching hospital. Sections 5 and 6 illustrate, respectively, how BIM supports modeling activity and how CIM supports the mapping to interconnect BIM to data. Section 7 illustrates the related work, followed by Section 8 which provides an evaluation of our approach. Section 9 presents our conclusions and plans for future research.

\section{Baseline}

We give a brief overview of managing organizational transformation and Business Intelligence, as well as the foundations of our Business Intelligence Model (BIM) and Conceptual Integration Model (CIM).

Organizational Transformation and Business Intelligence - Organizational transformation $[4,5]$ is a process through which low-performance organiza- 
tions change state and become strategically healthy. As described in [6], "strategically healthy organizations respond efficiently to change, anticipate change in a beneficial way, and lead change within their industries". Business Intelligence [7] can be a powerful enabler for such a strategic transformation in order to produce a high-performance organization. In particular, BI systems combine operational data with analytical tools to present complex and competitive information to planners and decision makers. In fact, as described in [8], BI is a process that includes two primary activities: getting data in and getting data out. The former involves moving data from a set of sources into an integrated data warehouse; the latterconsists of business users (and applications) accessing data from the data warehouse to perform enterprise reporting, OLAP querying, and predictive analysis. BIM supports getting data in activities by defining clear requirements that make explicit the information needed to evaluate strategies; and getting data out activities by presenting to the user an abstract view of the their business (in terms of goals, processes, resources, and other concepts) and its performance. On the other hand, CIM collects and integrates organization's data sources (therefore, it supports getting data in activities) and makes them available to BIM, and in turn, to the business users.

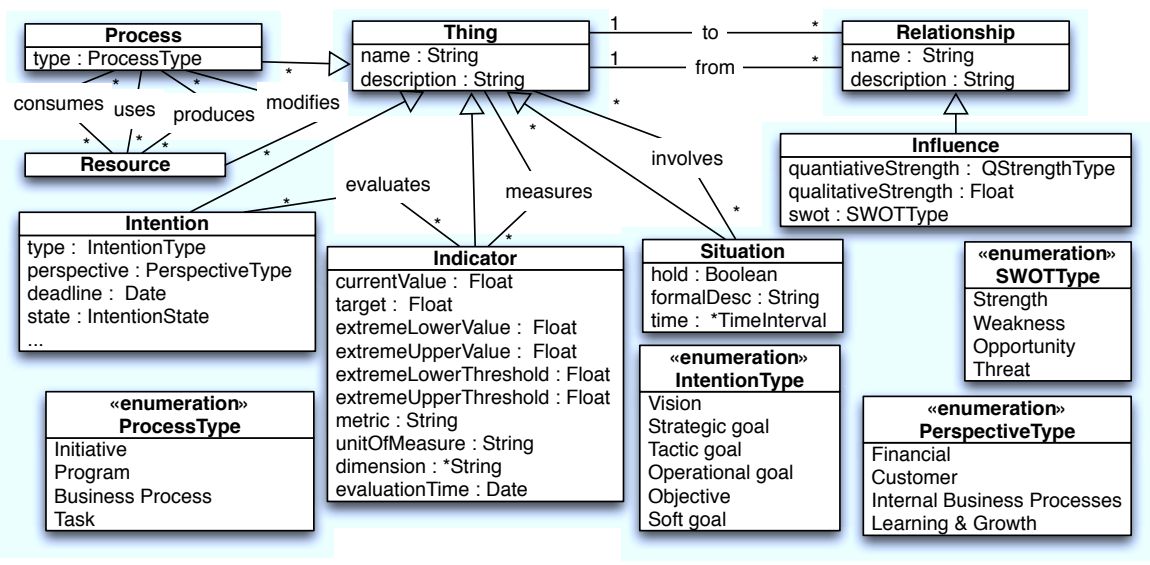

Fig. 1. A fragment of BIM.

The Business Intelligence Model - The Business Intelligence Model [9] allows business users to conceptualize their business operations and strategies using concepts that are familiar to them, including Actor, Directive, Intention, Event, Situation, Indicator, Influence, and Process. Figure 1 shows the fragment of BIM used in this paper (see [9] for details). BIM is drawn upon well-established concepts and practices in the business community, such as the Balanced Scorecard and Strategy Maps [10, 11], as well as techniques from conceptual modeling and enterprise modeling, such as metamodeling and goal modeling techniques. 
In particular, BIM can be used by business users to build a business schema of their strategies and operations and performance measures. Users can therefore query this business schema using familiar business concepts, to perform analysis on enterprise data, to track decisions and their impacts, or to explore alternate strategies for addressing problems. The business queries are translated through schema mappings into queries defined over databases and data warehouses, and the answers are translated back into business-level concepts. BIM works together with CIM to address such an issue and, in this paper, we show how such a connection is performed (in particular) for indicators.

The Conceptual Integration Model - A data warehouse is a repository of data that has been materialized for statistical and analytical purposes. Data warehouses are organized in multidimensional fashion, i.e., the basic data stored in fact tables are linked to various views or dimensions that help analyze the data in multiple ways. As in the relational model [12], there is an impedance mismatch between (business intelligence) applications accessing the data and data's physical storage. The problem is exacerbated by the fact that the underlying multidimensional data is physically organized for data access performance rather than to reflect the conceptual and business models that the data and business analysts have in mind.

To raise the level of abstraction and bridge the ever increasing gap existing between physical data warehouse schemas and conceptual multidimensional models, the Conceptual Integration Modeling (CIM) Framework was proposed [13]. The CIM Framework offers both design time and run time environments based on a CIM Visual Model (CVM), which provides two different views of a data warehouse: a conceptual model of the data (called CVL - Conceptual Visual Language) and a physical model of the data (called SVL - Store Visual Language). In other words, the CVL provides an abstract, high-level view of the data stored in the physical tables of the SVL. The representational gap between the CVL (conceptual) and the SVL (physical) models is filled by the MVL (Mapping Visual Language) consisting of correspondences (with optional value conditions) between attributes of entities in the CVL and the SVL models. The CIM tool can then compile these simple correspondences into complex views over the physical model that can be used to efficiently evaluate queries posed on the conceptual model.

\section{Managing the Strategic Initiative Lifecycle}

Enacting organizational transformation through the implementation of strategic initiatives is a well understood process $[4,10,11]$ that is taught in business schools. Changes to organizational intentions, processes, and resources are implemented and monitored in order to address a particular problem or opportunity. Figure 2 shows how the key elements of our framework integrate with and support the iterative lifecycle of a strategic initiative. The lifecycle is iterative because the organizational changes enacted by the initiative are refined and updated based on the feedback provided by indicators. The mapping framework 


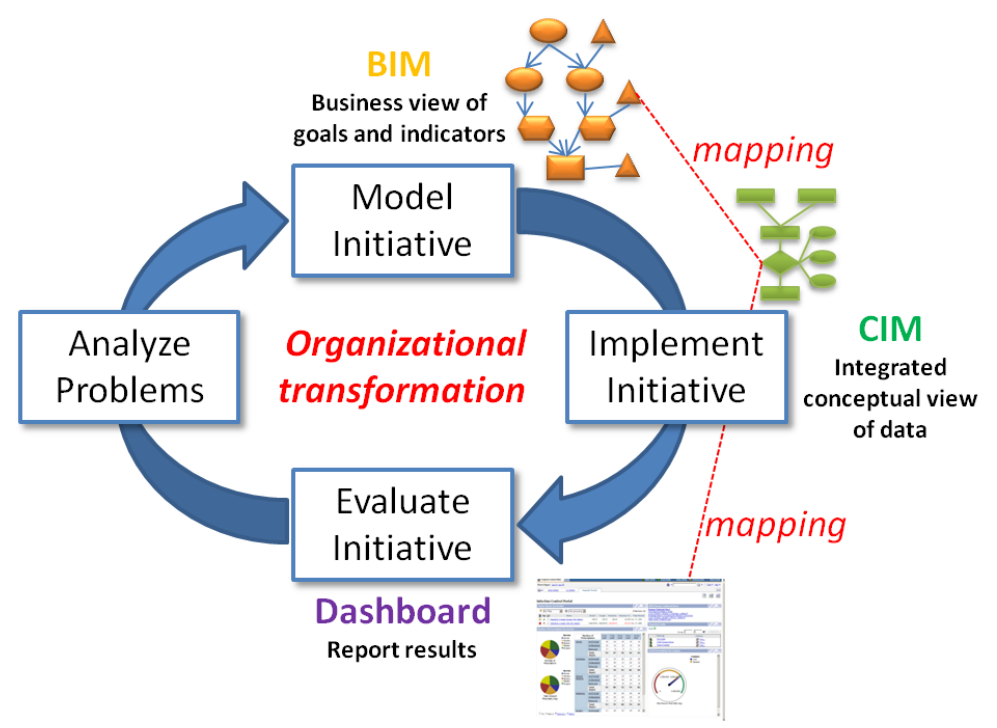

Fig. 2. Model-based management of strategic initiatives.

from BIM to CIM and from CIM to Dashboard facilitates implementation and maintenance of the initiative throughout its lifecycle.

Model Initiative: an initiative is modeled by the business analyst in all its aspects through the use of BIM. Strategic goals are defined and decomposed hierarchically until operational goals are reached. Business processes are described along with resources they use, consume and produce in order to achieve the hierarchy of goals. To evaluate the performance of the initiative, performance measures, i.e., key performance indicators, are created and associated to strategic goals, business processes, resources, actors, etc.

Implement Initiative: the initiative is implemented within the organization where business processes are executed and performed by employee, policies are enforced, resources are consumed and produced, and so on. In this phase, data are collected and integrated from a variety of applications, systems and documents into a data mart or a data warehouse. From such integrated view, CIM is used to obtain a corresponding conceptual representation which, in turn, is connected to BIM.

Evaluate Initiative: Performance measures are calculated from the collected data and are evaluated against the defined targets. Dashboards [14] are used to report such evaluations to the business users allowing for insight to reveal whether or not an actual value for a business's aspect deviates too far from a pre-defined target. Past trends and predictions can be also visualized.

Analyze Problems: Further analysis is performed on critical area identified in the previous phase to understand why an organization may or may not be on track to meet a specific target or objective. In this phase, operational information collected via monitoring is used to identify the causes of faults and errors as 
they occur, as well as to forecast performance levels and threats to operational stability. Discoveries made during analysis should help the management in planning next steps, set new (or adjust existing) expectations, and predict what may happen based on organization's decisions.

The organizational transformation cycle allows for a continuous improvement process in which feedback from the measurement system provides managers with the necessary information to make change or adjust business activities. The details of the framework are explained and demonstrated through the use of a case study in the next sections.

\section{Case Study: Reducing Antibiotic Resistant Infections}

We will explain and illustrate our model-based framework for managing organizational change using examples drawn from a strategic initiative currently underway at a large teaching hospital to Reduce Antibiotic Resistant Infections (RARI) by changing the way antibiotics are used. Increasingly, hospitals have been plagued with outbreaks of micro-organisms that are resistant to antibiotics, including Clostridium difficile (C.difficile), methicillin-resistant staphylococcus aureus (MRSA), and vancomycin resistant enterococcus (VRE). One reason for these outbreaks is the overuse of antibiotics, which selectively allows these organisms to thrive in an environment [15]. Antibiotics are also very expensive. They account for about $30 \%$ of a typical hospital's pharmacy budget [16]. Thus, overuse of antibiotics leads to increased morbidity in patients and excess cost.

The ultimate goal of the RARI initiative is to reduce the number of incidents of antibiotic resistant infections, but the focus of the initiative is to limit the amount and number of prescriptions for antibiotics deemed to be high risk. An education campaign for physicians will be created to change the type, amount and number (or percentage) of antibiotic prescriptions. Correct medication guidelines will be defined for antibiotic usage and it will be monitored with monthly and annual reporting of prescription rates (percentage, number, total amount, and duration) by service, location, physician, and antibiotic type. It is expected that there will be cost savings to the hospital both from fewer antibiotics used, and through a lower rate of incidents.

Enacting such a strategic initiative is a complex task both from a business point of view and a technology point of view. In particular, it is important to precisely define the indicators that will be used to monitor whether the goals of the initiative are being met and map this definition accurately and efficiently to the collection and reporting of the data used to measure the indicators. The data needed for the indicators must be integrated from many different data sources including the pharmacy records, administrative records that indicate where patients were located (bed, unit, campus) when the prescription was made, and for what service the prescribing physician was working. As well, individual departments within the hospital each have their own clinical information systems to classify which antibiotics in what amounts are appropriate for what diagnoses. 
An infection control dashboard was created for infection control analysts to monitor indicators and evaluate the effectiveness of the strategic initiative. It presented a dimensional view of indicators relevant to the initiative. The dimensional view allowed prescription usage to be broken down by time (hour, day, month, year), drug (drug category, drug type, drug brand), location (bed, nursing unit, campus) and by organization (physician, service, department). The indicators tracked were the number of antibiotic prescriptions, the percentage of antibiotic prescriptions (over all prescriptions), the average duration of antibiotic prescription (measured in hours), and the average and total amount of antibiotic prescription(s) (measured in milligrams for the entire duration of the prescription).

\section{Modeling Strategic Initiatives with BIM}

The set of primitives provided by BIM allows a business user to define a business schema representing the RARI initiative undertaken by the hospital. The initiative is modeled in terms of strategic goals, processes and resources, and is monitored by indicators to understand whether or not goals are met or to identify possible sources of problems. A complete description of how such schemas are built can be found in [17]; while, in the following sections, (part of) the business schema to define and monitor the RARI initiative is shown.

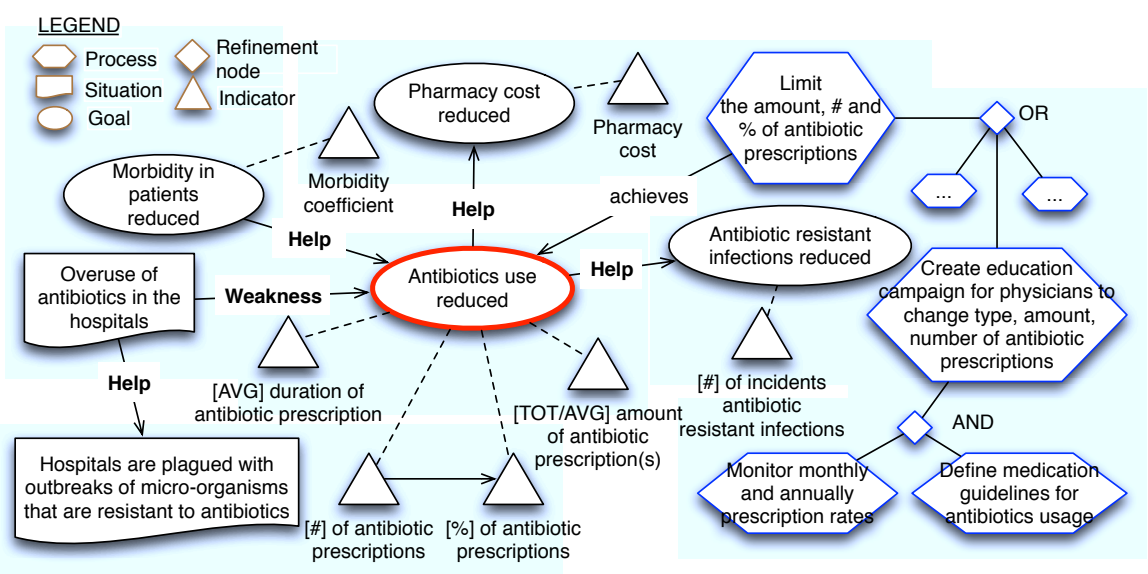

Fig. 3. Strategic goals.

Strategic Goals Definition - Figure 3 illustrates the high level strategic goals for the hospital and the RARI initiative. The BIM Intention primitive is used to represent the hospital's strategic goals ${ }^{1}$, while the Situation primitive is used ${ }^{1}$ The term Strategic goal is one of the values which can be assumed by the type
attribute associated to the Intention primitive (see Figure 1). 
to represent those partial states of the world which can positively or negatively influence such goals. For example, the Situation "Overuse of antibiotics in the hospital" undermines or weakens the hospital's goal to reduce the use of antibiotics. In the figure, the meaning of weakness and threat labels derives from SWOT analysis [18], in which the former represents an internal factor to the hospital that is harmful to achieving the goals while the latter is an external factor or condition which could do damage to the goals. To reason among goals and among situations, we use a qualitative (scale) contribution to characterize influence relationships among goals and among situations as is supported in GRL models [19]. For example, the "help" label in Figure 3 should be read as: a reduced use of antibiotics "helps" to reduce pharmacy cost (see Section 4) while a situation in which antibiotics are overused can favor (help) outbreaks of micro-organisms that are resistant to antibiotics.

Figure 3 also shows the RARI initiative and its decomposition. Due to space limitation, only one alternative is shown for its refinement, i.e., the education campaign creation, but more actions can be planned to reduce antibiotic resistant infections. The figure also shows a set of Indicators that are defined to monitor the impact of the initiative on the strategic goals. For simplicity, in the rest of the paper we will focus the analysis on the "Antibiotics use reduced" goal, but a similar analysis can be done for all the strategic goals in the figure, e.g, the "Antibiotic resistant infections reduced".

The Drug Treatment Process - Figure 4 describes the drug treatment process where antibiotics are prescribed. We can see that it is decomposed into the "Medication prescription" and "Medication administration" activities. In BIM, resources can be classified according to their nature; for example, we have information resources, human resources, capability/skill resources, etc. Moreover, BIM provides four relationships among processes (or activities) and resources, namely uses(p,r), consumes( $p, r)$, modifies $(p, r)$, and produces $(p, r)$. An in-depth description of resource classification and their relationships with processes can be found in $[9,17]$. For instance, in Figure 4, a prescription is produced by the "Medication process" by i) using information on patients and on the drugs available in the hospital, ii) using and consuming, respectively, skills and time of a doctor (this is the meaning of the the use/consume relationship associated to a human resource in the figure).

Notice how BIM allows to define indicators on processes and resources to monitor their performance with respect to intentions. Indeed, BIM helps to motivate why an indicator is needed (e.g, to evaluate antibiotics use reduction) and which aspect of business such an indicator must measure (e.g., the amount of antibiotic in a prescription). For example, in Figure 4, with respect to the RARI initiative, we need to concentrate on those indicators associated with the prescription resource since they monitor the doctor behavior ${ }^{2}$ the initiative aims to modify.

\footnotetext{
${ }^{2}$ In fact, the term prescription is commonly used to mean an order (from a doctor to a patient) to take certain medications, while we use the term drug dose to identify the actual medication's dose a nurse administrates to a patient.
} 


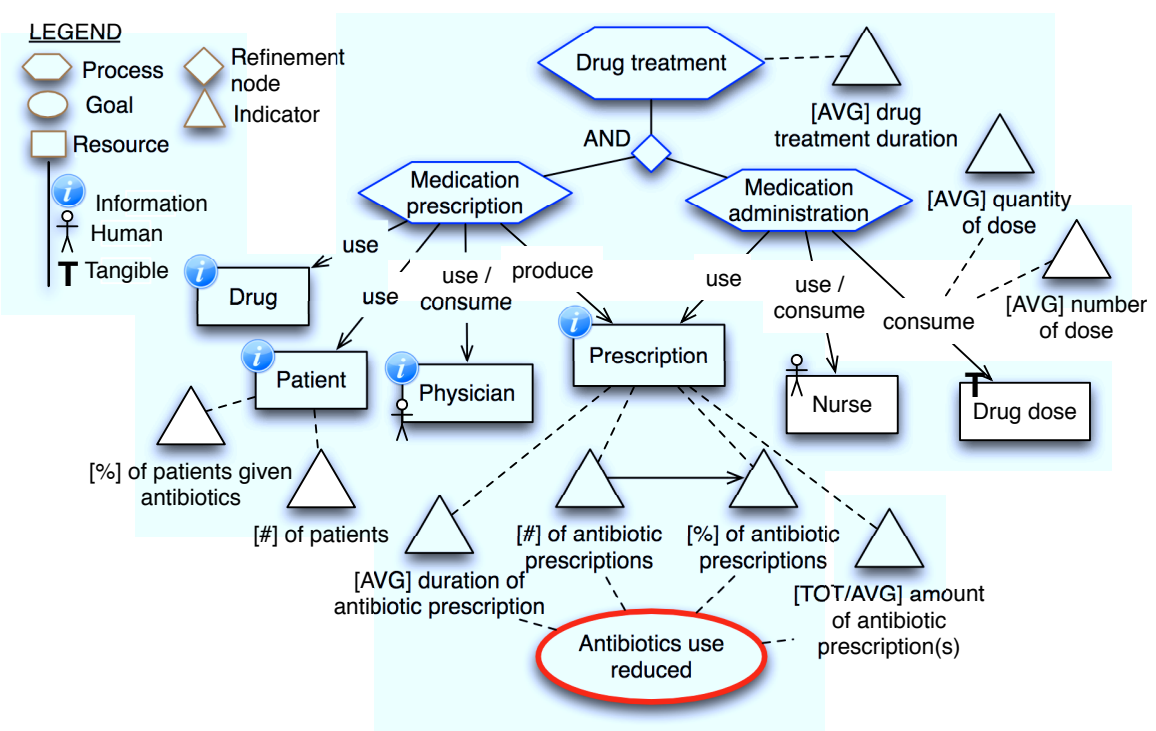

Fig. 4. The Drug treatment process.

In the following section, we show how BIM can be supported by CIM to feed indicators with data.

\section{Data Mappings in CIM and BIM}

Figure 5 shows a CIM model consisting of a CVL (on the left) and an SVL (on the right). Medication and Prescriber (shadowed rectangles) are CVL dimensions describing measures in the Prescription fact relationship (shadowed diamond). Non-shadowed rectangles (e.g., Drug, Physician) represent CVL levels in the dimensions. These levels are organized into hierarchies by parent-child relationships, which are drawn as edges between levels. For instance, the Prescriber hierarchy indicates that all physicians roll up to Unit, Campus and Service. The SVL is a UML-like representation of the relational data warehouse schema, containing relational table definitions, keys and referential integrity constraints. The left to right dashed arrows are part of the MVL and represent the correspondences between the models. For instance, the CVL Prescription fact relationship is physically stored in two different data warehouse tables: the SVL Prescription and Patient.

The CVL specification corresponds to what is increasingly called the semantic layer in industry. Such a layer liberates users from the low-level multidimensional intricacies and allows them to focus on a higher level of abstraction. For instance, the SVL model in the figure has normalized tables, which is not necessarily the best way to represent multidimensional entities in the conceptual view. 


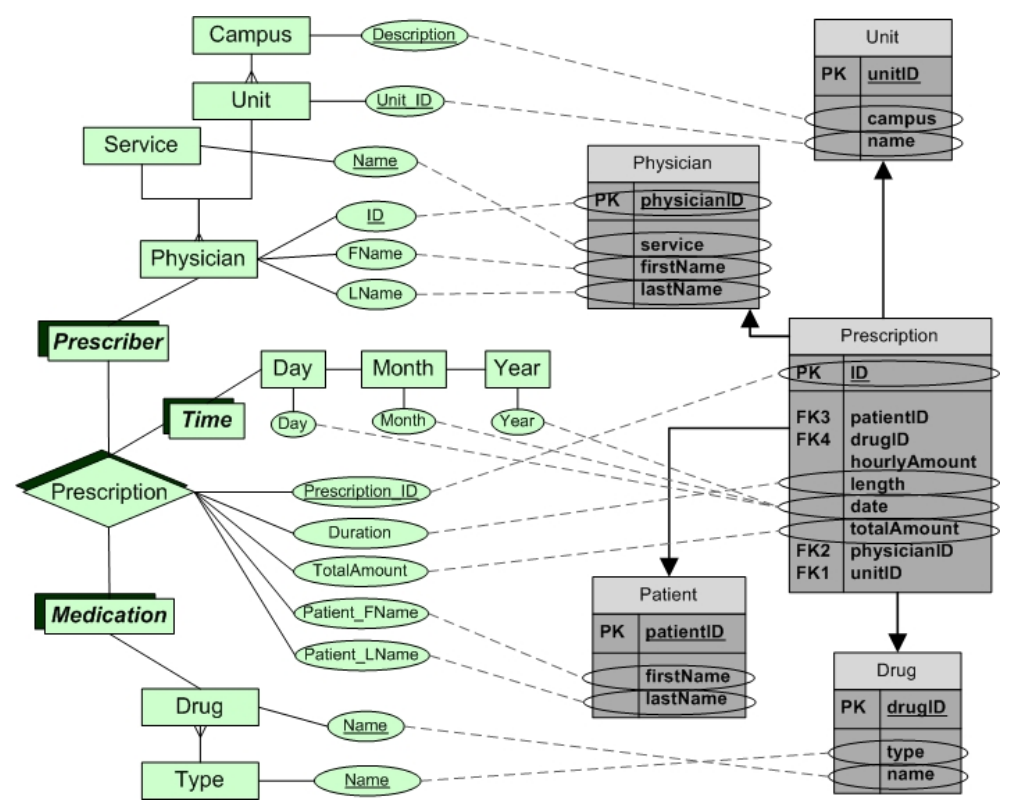

Fig. 5. CIM Visual Model for the medication prescription activity: CVL (left), SVL (right) and MVL (left-right dashed lines).

It is important to note that the only model that contains materialized data is the SVL; the CVL can access SVL data only through mappings. Interestingly, the user-defined correspondences that appear in the MVL are not sufficient for exchanging data from the SVL to the CVL - some data dependencies are lost by such simple attribute-to-attribute mappings. For data exchange, the CVL and SVL models are related by more complex mappings [20].

However, it is not practical for a high-level data analyst accustomed to deal only with the conceptual view of the data to come up with such complex view definitions in terms of the tables of the physical data warehouse. That is the reason why CIM requires from the user only very simple correspondences between attributes. Then, the CIM tool takes care of transparently compiling the user-defined correspondences into complex, fully-fledged, multidimensional mappings that can be used for query evaluation [21]. This is similar to the approach followed by EDM [12] for the relational setting.

A similar situation arises when trying to map a business model to an existing data warehouse with SVL, CVL and MVL already defined. In that situation, BIM entities can be related to a query expression (view) over the CVL, much in the same way CVL entities are mapped to views over the SVL. Such expressions can be complex multidimensional queries with aggregations and roll up functions. Again, writing these complex expressions is not practical for a business user. Instead, the business user draws simple correspondences between the models at hand, this time between BIM and CVL, and the BIM tool generates the 


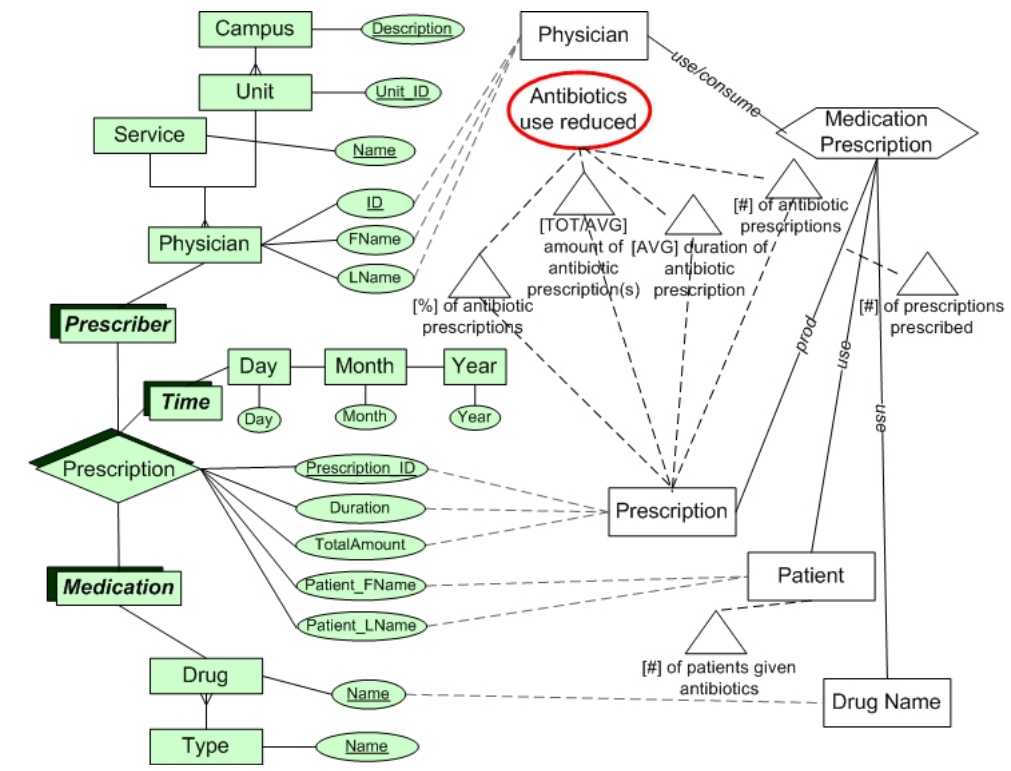

Fig. 6. BIM+CIM Visual Model for the medication prescription activity: CVL (left), BIM (right) and mappings (left-right dashed lines)

multidimensional CVL views representing the user's data requirements expressed in the correspondences.

Consider Figure 6. The CVL model on the left-hand side is the one from Figure 5. The BIM model on the right-hand side corresponds to the Medication Prescription activity of Figure 4. BIM entities have attributes that are not represented in the figure for simplicity - they happen to have the same names as the CVL attributes they are mapped to. For instance, there are four correspondences from the BIM Prescription resource to four attributes in the CVL Prescription fact relationship, i.e., Prescription_ID, Duration, Date and TotalAmount, which are also the names of the Prescription resource attributes. Moreover, for Indicators we have (hidden) information such as target, threshold, current value, etc., but also, more important for the mapping task, dimensions and levels to represent hierarchy ${ }^{3}$.

The BIM mapping compilation takes these correspondences and creates views over the CVL. For instance, BIM Prescription is mapped to a CVL view defined as V1=Prescription(Prescription_ID, Duration, Date, TotalAmount), basically a SELECT query in SQL. Every time the Prescription resource needs to pull data from CIM, view V1 is used.

Some other views involve roll up queries with aggregations, i.e., the views to feed BIM indicators. For instance, for the following BIM indicators we have:

\footnotetext{
${ }^{3}$ The possible dimensions (and levels) available for an indicator are elicited by the CVL fact table with which it is associated, e.g., the dimensions for [\#] of antibiotic prescriptions are "Prescriber" and "Medication".
} 
- [\#] of antibiotic prescriptions: The actual value for the indicator is obtained by a query that aggregates the number of instances that appear in the CVL Prescription fact table which have a value equals to "antibiotic" for the Type.Name attribute in the Medication dimension.

- [\%] of antibiotic prescriptions: The actual value for the indicator is obtained by the value of [\#] of antibiotic prescriptions divided by a query that aggregates the number of instances that appear in the CVL Prescription fact table, all multiplied by 100 .

- [TOT/AVG] amount of antibiotic prescription(s): The actual value for the indicator is obtained by a query that aggregates the amounts that appear in the CVL attribute Prescription.TotalAmount for all those corresponding to Type.Name= "antibiotic" on the Medication dimension. If the average is requested, the above value is divided by the [\#] of antibiotic prescriptions.

- [AVG] duration of antibiotic prescription: The actual value for the indicator is obtained by a query that aggregates the durations that appear in the CVL attribute Prescription. Duration for all those corresponding to Type.Name= "antibiotic" on the Medication dimension, and divides it by the [\#] of antibiotic prescriptions.

As explained above, the current values for these indicators can be drilleddown using dimensions and levels defined in the BIM Indicator. Indeed, when a drill-down action is performed, a corresponding query is performed on the CVL. For example, a BIM user can desire to have [\#] of antibiotic prescriptions prescribed by a Physician named "John Smith". In such a case the above query is reformulated considering the Prescriber dimension with Physician.FName= "John" and Physician.LName="Smith".

\section{Related Work}

In the literature, different approaches from goal-oriented requirements engineering, e.g., [22, 23], combine intentional and social concepts to model organization strategies and their elements (e.g., actors, resources, and processes). Other works have also extended $i^{*}[22]$ and related frameworks (e.g., URN [23]) towards enterprise and business modeling, e.g., [24]. A recent extension of URN includes indicators [2], but does not address the question of how to link the business level view to technology. The BIM aims to unify various modeling concepts into a coherent framework with reasoning support and connection to enterprise data, built upon a firm conceptual modeling foundation. In particular, with respect to the above works, BIM includes (among others): the notion of influence which is adopted from influence diagrams [25], a well-known and accepted decision analysis technique; SWOT analysis concepts [18] (strengths, weaknesses, opportunities, and threats) and others which are adopted from OMG's Business Motivation Model standard [26]; and support for Balanced Scorecard and Strategy Maps $[10,11]$. 
Moreover, BIM's concepts are formalized through metamodeling in terms of abstract concepts such as Thing, Object, Proposition, Entity, and Relationship, taking inspiration from the DOLCE [27] ontology.

A number of conceptual multidimensional schemas for warehouse modeling have been proposed over the years (see [28] and references therein). Such approaches are mainly proposals for modeling languages that are part of data warehouse design methodologies. By contrast, CIM [13] provides a run-time environment that allows a user to pose queries and do business analytics at conceptual and business levels.

On the industry side, two major vendors of business analytics solutions (namely SAP Business Objects and IBM Cognos) provide proprietary conceptual levels that they call "semantic layers". SAP Business Objects' semantic layer [29], called a Universe, is a business representation of an organization's data asset (i.e., data warehouse as well as transactional databases). IBM Cognos' semantic layer, Framework Manager [14], is similar to SAP's Universes and works according to the same principles.

In contrast to these approaches, the EDM Framework [30] provides a querying and programming platform that raises the level of abstraction from the logical relational data level to Peter Chen's Entiry-Relationship (ER) conceptual level [31]. EDM consists of a conceptual model, a relational database schema, mappings between them and a query language (Entity SQL - eSQL), over the conceptual model. A compiler generates the mapping information in the form of eSQL views that express ER constructs in terms of relational tables. Unlike CIM, which deals with the multidimensional data model, EDM deals with the classical relational data model.

\section{Evaluation}

The cyclic approach of Model, Implement, Evaluate, Analyze is not new. It is a classical approach to managing strategic initiatives that is taught in business schools, and is carried out by organizations around the world. However, the gap between the business view, the technical data view, and the results reported is quite large and is bridged largely in a manual, ad hoc fashion. Our approach leverages models to structure, systematize and automate the process, and provides analysts with novel tools that they do not currently have:

- A structured representation of the business view of a strategic initiative, which links goals to tasks that accomplish them and indicators that measure them.

- A conceptual view of data that collects the required data from disparate data sources across the organization in order to compute the indicators and report on them in a dashboard.

- A systematic approach to mapping from business view to conceptual view to dashboard.

- An opportunity for tool-based support for analysts to design, implement, and manage strategic initiatives. 
- Formal mappings that ensure that changes to goals indicators, dashboards, and data sources can be flexibly accommodated, facilitating maintenance.

- Better support in the cognitive gaps between the business and technological points of view, which allows savings in terms of time, accuracy, and other qualities during the implementation phase.

\section{Conclusions and Future Work}

We have presented a model-based framework for bridging the business and technological levels within organizations. The workings of the framework are demonstrated through a case study that involves managing the strategic initiative lifecycle at a teaching hospital implementing an initiative intended to reduce antibiotic resistant infections. The case study demonstrates how the framework works, but also how it can help bridge cognitive gaps and reduce the need for manual processing.

Our plans for future work include fleshing out the framework and supporting it with tools that automate or semi-automate some of the implementation tasks. With this aim, the concepts of flexibility and adaptability defined in [32] will be investigated and applied to our approach to: i) satisfy the changing data analysis requirements of business users; and ii) cope with changes in local data sources. This will allow for the delivery of timely and accurate BI to business users.

Acknowledgments. This work has been supported by the Business Intelligence Network (BIN) and the Natural Sciences and Engineering Research Council of Canada. We are grateful to Eric Yu, Iluju Kiringa and many other colleagues for useful discussions and suggestions that helped shape this work.

\section{References}

1. Vonderheide-Liem, D.N., Pate, B.: Applying quality methodologies to improve healthcare: Six sigma, lean thinking, balanced scorecard, and more. HCPro, Inc. (2004)

2. Pourshahid, A., Amyot, D., Peyton, L., Ghanavati, S., Chen, P., Weiss, M., Forster, A.J.: Business process management with the User Requirements Notation. Electronic Commerce Research 9(4) (2009) 269-316

3. Kronz, A.: Managing of process key performance indicators as part of the aris methodology. In: Corporate Performance Management. Springer (2006) 31-44

4. Nadler, D.A., Shaw, R.B., Walton, A.E., Associates: Discontinuous change: Leading organizational transformation. JOSSEY-BASS, An Imprint of WILEY (1994)

5. Galliers, R.D., Baets, W.R.J., eds.: Information technology and organizational transformation: Innovation for the 21st century organization. John Wiley Series in Information Systems (1998)

6. Burgin, A.L., Koss, E.: Transformation to High Performance. A journey in organizational learning. Report No. 823. (Summer 1993)

7. Negash, S.: Business intelligence. CAIS 13(15) (2004)

8. Watson, H.J., Wixom, B.H.: The current state of business intelligence. Computer 40 (September 2007) 96-99 
9. Barone, D., Mylopoulos, J., Jiang, L., Amyot, D.: Business Intelligence Model, version 1.0. Technical Report CSRG-607 (ftp://ftp.cs.toronto.edu/csri-technicalreports/INDEX.html), University of Toronto (March 2010)

10. Kaplan, R.S., Norton, D.P.: Balanced Scorecard: translating strategy into action. Harvard Business School Press (1996)

11. Kaplan, R.S., Norton, D.P.: Strategy maps: Converting intangible assets into tangible outcomes. Harvard Business School Press (2004)

12. Melnik, S., Adya, A., Bernstein, P.A.: Compiling mappings to bridge applications and databases. In: SIGMOD, New York, NY, USA, ACM (2007) 461-472

13. Rizzolo, F., Kiringa, I., Pottinger, R., Wong, K.: The conceptual integration modeling framework: Abstracting from the multidimensional. arXiv:1009.0255 (2010)

14. Volitich, D.: IBM Cognos 8 Business Intelligence: The Official Guide. McGraw-Hill (2008)

15. Mazzeo, F., Capuano, A., Avolio, A., Filippelli, A., Rossi, F.: Hospital-based intensive monitoring of antibiotic-induced adverse events in a university hospital. Pharmacological Research 51(3) (2005) 269-274

16. Salama, S., Rotstein, C., Mandell, L.: A multidisciplinary hospital-based antimicrobial use program: Impact on hospital pharmacy expenditures and drug use. Can. J. Infect. Dis. 7(2) (1996) 104-109

17. Barone, D., Yu, E., Won, J., Jiang, L., Mylopoulos, J.: Enterprise modeling for business intelligence. PoEM (2010)

18. Dealtry, T.R.: Dynamic SWOT Analysis. Dynamic SWOT Associates (1994)

19. Amyot, D., Horkoff, J., Gross, D., Mussbacher, G.: A lightweight GRL profile for i* modeling. In: ER Workshops, Berlin, Heidelberg, Springer-Verlag (2009) 254-264

20. Lenzerini, M.: Data integration: a theoretical perspective. In: PODS, New York, NY, USA (2002) 233-246

21. Nargesian, F., Rizzolo, F., Kiringa, I., Pottinger, R.: Bridging decision applications and multidimensional databases. SITE, University of Ottawa (2010)

22. Yu, E.: Towards modelling and reasoning support for early-phase requirements engineering. In: RE '97, Washington, USA (1997)

23. International Telecommunication Union: Recommendation Z.151 (11/08): User Requirements Notation (URN) - Language definition. http://www.itu.int/rec/TREC-Z.151/en.

24. Andersson, B., Johannesson, P., Zdravkovic, J.: Aligning goals and services through goal and business modelling. Inf. Syst. E-Business Management 7(2) (2009)

25. Howard, R., Matheson, J.: Influence diagrams. Readings on the Principles and Applications of Decision Analysis, Vol. II (1984)

26. Business Rules Group: The Business Motivation Model: Business Governance in a Volatile World. http://www.businessrulesgroup.org/bmm.shtml (2007) Ver. 1.3.

27. Gangemi, A., Guarino, N., Masolo, C., Oltramari, A., Schneider, L.: Sweetening ontologies with DOLCE. In: EKAW 2002, London, UK, Springer-Verlag (2002)

28. Malinowski, E., Zimanyi, E.: Advanced Data Warehouse Design: From Coventional to Spatial and Temporal Applications. Springer, Berlin (2008)

29. Howson, C.: BusinessObjects XI (Release 2): The Complete Reference. McGrawHill (2006)

30. Adya, A., Blakeley, J.A., Melnik, S., Muralidhar, S., the ADO.NET Team: Anatomy of the ADO.NET Entity Framework. SIGMOD (2007)

31. Chen, P.P.S.: The entity-relationship model - toward a unified view of data. ACM Trans. Database Syst. 1(1) (1976) 9-36

32. Cheung, W., Babin, G.: A metadatabase-enabled executive information system (part A): a flexible and adaptable architecture. DSS 42 (2006) 1589-1598 